Inclusion and rigor in qualitative autism research: A response to Van Schalkwyk and Dewinter (2020)

\author{
Laura Crane $^{1}$, Abby Sesterka ${ }^{2}$ and Jacquiline den Houting ${ }^{3,4}$ \\ ${ }^{1}$ Centre for Research in Autism and Education (CRAE), University College London, UK \\ ${ }^{2}$ College of Education, Psychology and Social Work, Flinders University, Australia \\ ${ }^{3}$ Macquarie School of Education, Macquarie University, Australia \\ ${ }^{4}$ Cooperative Research Centre for Living with Autism, Australia
}

Correspondence to: Laura Crane

Centre for Research in Autism and Education (CRAE)

UCL Institute of Education, University College London

London, WC1H 0NU, United Kingdom

L.Crane@ucl.ac.uk

Running Head: Qualitative autism research 


\section{Inclusion and rigor in qualitative autism research: A response to Van Schalkwyk and Dewinter (2020)}

In their recent editorial in the Journal of Autism and Developmental Disorders (JADD), Van Schalkwyk and Dewinter (2020) rightly outline the value of qualitative research in better understanding the lived experiences of autistic people and their families and amplifying the voices of the real experts on autism. This is crucially important. Autism research has traditionally been conducted on, about, or for autistic people and their families, without the involvement of those with insider expertise (Pellicano et al., 2014). Encouragingly—and largely as a result of community advocacy and the growing number of autistic scholars - this has started to change. Autistic people and their families are beginning to play key roles in research priority setting (e.g., Australian Autism Research Council, 2019; Autistica, 2016; Frazier et al., 2018; Pellicano et al., 2014), and in the design and conduct of research (e.g., Nicolaidis et al., 2012). Qualitative research can complement such initiatives, providing an opportunity for the voices of autistic people and their families to be heard —in depth and in their own words—allowing unique insights into their experiences.

We very much appreciate the need for Van Schalkwyk and Dewinter's (2020) editorial on qualitative autism research in JADD. With funders and advocates encouraging the inclusion of community voices in research (e.g., Cooperative Research Centre for Living with Autism, 2016; Nicolaidis, 2012; Warner et al., 2019), more autism researchers are making a shift from purely quantitative research to mixed-methods and/or qualitative studies, often without any substantial qualitative training or experience. As such, some of the qualitative studies published in autism-specific journals are perhaps yet to reach the standard that would typically be expected for qualitative research in other disciplines.

We are then faced with the need to foster autism researchers' interest in and enthusiasm for qualitative research, while also maintaining the high standards of research that autistic people deserve. Systematic reviews of qualitative autism research (e.g., Corcoran et al., 2015; Singh \& Bunyak, 2019) indicate that much of the qualitative literature in this field has, to date, been published outside of the major autism journals. As such, caution must be exercised to ensure that qualitative scholars are not excluded from the dominant discourse, and gatekeeping practices should be discouraged. We are concerned that Van Schalkwyk and Dewinter (2020) risk exacerbating these issues with their proposed guidelines for the conduct of qualitative autism research (see Table 1 of their editorial for details). Having 
considered the proposed guidelines from our respective positions as an established non-autistic researcher (LC) and Autistic early career researchers (AS, JdH), we have identified three key elements that we wish to comment on.

First, Van Schalkwyk and Dewinter (2020) detail the methods that they feel are appropriate for high quality qualitative studies published in JADD: semi-structured interviews, focus groups or observational studies. Whilst these methods are commonly employed in qualitative work, this guidance does not reflect the unique context of research with autistic people and their families. Guidelines on the inclusion of autistic people as research participants, for example, emphasize the importance of offering "multiple modes of participation to maximize the inclusion of autistic people with differing strengths and needs" (Nicolaidis et al., 2019, p. 2014). Nicolaidis et al. make specific reference to the utility of asynchronous modes of communication and/or written forms of communication as research tools. Yet, Van Schalkwyk and Dewinter (2020, p. 2281) suggest that written responses from surveys are "often not a sufficiently robust qualitative method"; a statement seemingly at odds with Nicolaidis et al.'s best practice guidance for conducting respectful, accessible, and methodologically sound research with the autistic community. Communication differences aside, online qualitative surveys may also serve to facilitate research participation of other stakeholder groups, including the parents and carers of autistic people. These community members are often time-poor (Ludlow et al., 2012), and the burden of participation in an interview or focus group may prove to be a considerable barrier. Additionally, we note that many groups within the autism community—for example, autistic people with co-occurring intellectual disability, and those who are Black, Indigenous, and/or People of Color—are significantly underrepresented in research (Bennett \& Hodgson, 2017; Hilton et al., 2010; Russell et al., 2019). Limiting the data collection methods employed in our research may have the unintended consequence of further excluding these underrepresented groups (Lourenco \& Tasimi, 2020). In contrast, flexible and inclusive methods of qualitative data collection may encourage much needed diversity in autism research.

Second, we commend Van Schalkwyk and Dewinter (2020, p. 2281) for highlighting the need for qualitative research to "involve stakeholders in some aspect of the research design and analysis". Indeed, research conducted within a participatory framework tends to align more closely with the priorities of the autistic community, have better translation to practice, and yield improved outcomes for autistic people and their families (e.g., FletcherWatson et al., 2019). In light of this, we suggest that autistic involvement in the research process should extend far 
beyond qualitative studies, to include more basic autism science (Pellicano, 2020), especially since community dissatisfaction centers primarily around research not traditionally conducted using qualitative methods (e.g., research on biology, brain and cognition; see Pellicano et al., 2014). We also recommend that the involvement of stakeholders should, ideally, span the entire research process (as per Nicolaidis et al., 2011); and not be limited to "some aspect of the research design and analysis", as Van Schalkwyk and Dewinter (2020, p.2281) state. To truly highlight the value of participatory autism research, we suggest that JADD should consider extending this guideline to all research published in the journal, not only studies employing qualitative methods.

Third, Van Schalkwyk and Dewinter (2020) make very specific recommendations for improving the standards of qualitative autism research. These include a minimum sample size of at least 20 participants (in the absence of a strong theoretical justification for a smaller sample), and a requirement that data from one sample/project should always be reported in a single manuscript (as opposed to publishing detailed findings across several papers). While specific guidelines of this type are commonplace in quantitative research, we argue that qualitative research requires more flexibility and care must be taken to avoid imposing quantitative values onto qualitative practice. Indeed, we are concerned that the list of relatively rigid guidelines that Van Schalkwyk and Dewinter (2020) have proposed may encourage a "count-by-numbers" approach to qualitative research, rather than the reflexive and nuanced perspective required for rigorous and high-quality qualitative research. While discussion of the nuts and bolts of methods and methodology is useful and pragmatic, it is worth remembering that the core differences between quantitative and qualitative approaches derive from different traditions of scientific philosophy; the positioning of the researcher in relation to the research lies at the heart of this philosophical discussion (Denzin \& Lincoln, 1994; Smith, 1983).

In conclusion, we wholeheartedly agree with Van Schalkwyk and Dewinter (2020) that the field needs quality qualitative research, and very much applaud initiatives to encourage the submission of qualitative research to JADD as a means by which to enhance and progress the body of scholarship, and to draw benefit from the perspectives of qualitative researchers. Yet rather than relying solely on these guidelines, we would encourage those new to qualitative approaches to undertake qualitative research training, and develop familiarity with the various ontologies and epistemologies that underpin qualitative methods. Simply following Van Schalkwyk and Dewinter's (2020) guidelines might serve to elevate a previously poor-quality qualitative evidence base to adequacy but, to produce the 
high-quality qualitative research that the autistic community deserves, a deeper level of philosophical understanding is essential. 


\section{References}

Australian Autism Research Council (2019). Australian Autism Research Council: 2019 Research Priorities. https://www.autismcrc.com.au/sites/default/files/2019-

10/AARC\%202019\%20Autism\%20Research\%20Priorities.pdf

Autistica (2016). Top 10 Research Priorities. https://www.autistica.org.uk/downloads/files/Autism-Top-10-YourPriorities-for-Autism-Research.pdf

Bennett, M., \& Hodgson, V. (2017). The Missing Voices of Indigenous Australians with Autism in Research. Autism., 21(1), 122-123.

Cooperative Research Centre for Living with Autism. (2016). Inclusive Research Practice Guides and Checklists for Autism Spectrum Research: Version 2. Brisbane, Australia: Autism CRC Ltd.

Corcoran, J., Berry, A., \& Hill, S. (2015). The Lived Experience of US Parents of Children with Autism Spectrum Disorders: A Systematic Review and Meta-synthesis. Journal of Intellectual Disabilities, 19(4), 356-366.

Denzin, N., \& Lincoln, Y. (1994). Introduction: Entering the Field of Qualitative Research. In N. Denzin \& Y. Lincoln (Eds.), The SAGE Handbook of Qualitative Research (3rd ed., pp. 1-17). Thousand Oaks: SAGE.

Fletcher-Watson, S., Adams, J., Brook, K., Charman, T., Crane, L., Cusack, J., Leekham, S., Milton, D., Parr, J.R., \& Pellicano, E. (2019). Making the Future Together: Shaping Autism Research Through Meaningful Participation. Autism, 23(4), 943-953.

Frazier, T. W., Dawson, G., Murray, D., Shih, A., Sachs, J. S., \& Geiger, A. (2018). Brief Report: A Survey of Autism Research Priorities Across a Diverse Community of Stakeholders. Journal of Autism and Developmental Disorders, 48(11), 3965-3971.

Hilton, C. L., Fitzgerald, R. T., Jackson, K. M., Maxim, R. A., Bosworth, C. C., Shattuck, P. T., Geshwind, G. H., \& Constantino, J. N. (2010). Brief report: Under-representation of African Americans in Autism Genetic Research: A Rationale for Inclusion of Subjects Representing Diverse Family Structures. Journal of Autism and Developmental Disorders, 40(5), 633-639. 
Lourenco, S.F. \& Tasimi, A. (2020). No Participant Left Behind: Conducting Science During COVID-19. Trends in Cognitive Sciences, 24(8), 583-584.

Ludlow, A., Skelly, C., \& Rohleder, P. (2012). Challenges Faced by Parents of Children Diagnosed with Autism Spectrum Disorder. Journal of Health Psychology, 17(5), 702-711.

Nicolaidis, C. (2019). What Does Inclusion Mean in Research and Scholarship on Autism in Adulthood? Autism in Adulthood, 1(2), 79-81.

Nicolaidis, C., Raymaker, D., McDonald, K., Dern, S., Ashkenazy, E., Boisclair, C., Robertson, S., \& Baggs, A. (2011). Collaboration Strategies in Nontraditional Community-based Participatory Research Partnerships: Lessons from an Academic-Community Partnership with Autistic Self-advocates. Progress in Community Health Partnerships: Research, Education, and Action, 5(2), 143-150.

Pellicano, E. (2020). Commentary: Broadening the research remit of participatory methods in autism science - a commentary on Happé and Frith (2020). Journal of Child Psychology and Psychiatry, 61, 233-235.

Pellicano, E., Dinsmore, A., \& Charman, T. (2014). What Should Autism Research Focus Upon? Community Views and Priorities from the United Kingdom. Autism, 18(7), 756-770.

Russell, G., Mandy, W., Elliott, D., White, R., Pittwood, T., \& Ford, T. (2019). Selection Bias on Intellectual Ability in Autism Research: A Cross-sectional Review and Meta-analysis. Molecular Autism, 10, 9.

Singh, J. S., \& Bunyak, G. (2019). Autism Disparities: A Systematic Review and Meta-ethnography of Qualitative Research. Qualitative Health Research, 29(6), 796-808.

Smith, J. K. (1983). Quantitative Versus Qualitative Research: An Attempt to Clarify the Issue. Educational Researcher, 12(3), 6-13.

Van Schalkwyk, G. I., Dewinter, J. (2020). Qualitative Research in the Journal of Autism and Developmental Disorders. Journal of Autism and Developmental Disorders, 50, 2280-2282.

Warner, G., Parr, J. R., \& Cusack, J. (2019). Workshop Report: Establishing Priority Research Areas to Improve the Physical Health and Well-being of Autistic Adults and Older People. Autism in Adulthood, 1(1), 20-26. 


\begin{abstract}
Author note
Laura Crane: Centre for Research in Autism and Education (CRAE), UCL Institute of Education, UK; Abby Sesterka, College of Education, Psychology and Social Work, Flinders University, Australia; Jacquiline den Houting: Macquarie School of Education, Macquarie University, and Cooperative Research Centre for Living with Autism, Australia.
\end{abstract}

The authors are grateful to Professor Liz Pellicano, for her helpful comments on a draft of this article.

Correspondence concerning this article should be addressed to Dr Laura Crane, Centre for Research in Autism and Education (CRAE), UCL Institute of Education, University College London, 55-59 Gordon Square, London, WC1H 0NU, UK. +44(0)2073315141 L.Crane@ucl.ac.uk 